\title{
1 Introduction
}

You're sweating, you haven't slept properly in days, and you're pretty sure that you've been talking to yourself. Your search history is an endless stream of forums and reviews, and you've discovered that against all odds you're able to carry multiple completely opposing opinions in your head at the same time. You're pretty sure that you're about to lose it completely, possibly in a public place. You're scared. (Power \& Parker 2015)

This phenomenon has a name: 'GAS'. The abbreviation stands for 'Gear Acquisition Syndrome', a term that goes back to Steely Dan's guitar player Walter Becker, who wrote an editorial for the American Guitar Player magazine in 1996. Becker first contemplated the 'Guitar Acquisition Syndrome', a phenomenon he observed in the Los Angeles music scene and suspected many of the magazine's readers also to have:

I have decided to break my long standing editorial silence to draw the attention of the musical community at large and guitar players and guitar owners in particular to a grave situation whose tragic dimension is constantly expanding and is in fact threatening to engulf us all. Picture this: ... I'm working at a studio in town with another well known session cat who has had roughly the same readily identifiable and winning sound for the last twelve years or so-but I've noticed that he never shows up for a call with the same guitar twice-true, they all sound about the same but for some reason these excellent sounding (and looking) axes are constantly falling out of favor and being replaced by sonically indistinguishable ones - and further probing reveals that each one of these guitars has been extensively modified and remodified using the latest space age ... materials and techniques ..., only to be rejected and discarded AFTER TWO WEEKS OR LESS - What's up with these guys? It's called G.A.S.-Guitar Acquisition Syndrome. You undoubtedly know someone who has it. Reading this rag, you probably have it yourself. Or will have it someday soon or would like to have it. ... How many Strats [Stratocaster guitars] do you need to be happy? How many Strat copies, each extensively modified to be able to produce the variations in tone that once would have required maybe four different guitars? How many knobs and switches does that Strat need? ... The horror stories could fill this whole magazine (not a bad idea). (Becker 1996)

Becker expresses his unease with the scene's common practice of changing and modifying instruments unnecessarily with a wink. His intention for the editorial was to raise awareness of this practice being a widespread issue amongst musicians. GAS became a familiar acronym and eventually changed from 'Guitar Acquisition Syndrome' to 'Gear Acquisition Syndrome' because other musicians showed similar tendencies to guitar players.

Although acquiring musical instruments can indeed become an addiction, like any other form of compulsive collecting, GAS is usually not a 'clinical condition'. 
It is rather a cultural phenomenon which leads those affected to joke about themselves. In their view, GAS describes the unrelenting but harmless urge, triggered by the endless search for the 'magic tone', to buy and own gear as an anticipated catalyst of creative energy and bringer of happiness (Diiorio 2016). Humorous illustrations and discussions in musicians' boards can be found everywhere on the Internet, and GAS merchandise is sold online and in music stores. Video platforms showcase musicians' precious instrument collections, and the website www.guitaracquisitionsyndrome.com presents documentary films of most 'serious' cases. Although not directly related to GAS, it is worth mentioning that the German music instrument retailer Thomann (2019) hosts an annual summer camp as part of its 'Gearhead University'. YouTubers get the opportunity to review each piece of equipment in Thomann's warehouse in a small village in southern Germany, document their experiences on video and share them online with fellow musicians. This marketing strategy celebrates music gear for its own sake and takes the opportunity to influence the large community of subscribers to these video channels in their purchasing decisions. Furthermore, numerous blogs, online articles (Kwisses 2015; Leonhardt 2015; Power \& Parker 2015; Robair 2015) and even a video documentary (Diiorio 2016) are dedicated to GAS. Contrary to its omnipresence on the Internet, only a few print media discuss GAS. With Jay Wright's (2006) GAS: Living with Guitar Acquisition Syndrome, there is only one book on this topic. Even though being of journalistic nature, the book nevertheless provides rich qualitative data from interviews with 200 guitarists from 23 countries aged between 18 and 68 years.

The Merriam Webster (2019) dictionary defines a syndrome either as a 'group of signs and symptoms that occur together and characterize a particular abnormality or condition' or as a 'set of concurrent things (such as emotions or actions) that usually form an identifiable pattern'. In this book, the Gear Acquisition Syndrome will not be studied primarily as a compulsive disorder or addiction in the pathological sense. We instead understand it as a pronounced interest in music equipment, combined with a salient desire to acquire and possess certain items of gear. For those affected, the urge to make new purchases can be emotional. Sometimes, when triggered by watching a music video or reading a music magazine, it is short-lived and disappears within hours. At other times, the urge is longstanding, causing actions such as researching music equipment, testing gear in music stores and selling currently owned instruments or other possessions to finance a new purchase. In this study, we focus on the longstanding or recurrent disposition of the syndrome since the short-lived urge seems less significant. As Becker's (1996) editorial suggests, few musicians reach a point in their amateur or professional careers where they are entirely and indefinitely satisfied with their setup. Some musicians sell or trade instruments to make room for new ones, but others do accumulate equipment. There are rumours about impressive instrument collections like the ones of Scorpions guitarist Matthias Jabs with about 400 guitars, Aerosmith's Joe Perry with 600 guitars 
and the Rolling Stones' Keith Richards with allegedly about 3,000 guitars (Backhaus 2015; Legge 2011). Given the variety of forms that GAS can take, we expect it to involve diverse cultural practices. Those could vary due to various levels of professionalism, age and capital, gender, the type of musical instrument played, band membership and engagement with social media and other online platforms. These different practices and social contexts potentially spark, increase and maintain interest in music gear.

At first glance, GAS appears to be particularly pronounced among musicians of instruments whose sound production is technologically influenced, which we take as a starting point for further considerations. Reassuring is that popular music scholars have emphasised the central role of technology for popular music cultures. As Théberge (2001:3) rightly claims, '[a]ny discussion of the role of technology in popular music should begin with a simple premise: without electronic technology, popular music in the twenty-first century is unthinkable'. However, a closer look at the body of research reveals that most attention regarding music-related technologies has been paid to music production and the reception of records or other media. Since the late 1960s, researchers have advocated the recognition of the record as the primary medium of popular music (Belz 1969; Clarke 1983; Gracyk 1996; Zak 2001) and provided historical accounts of the development of recording technology and its creative use (Cunningham 1996; Moorefield 2010; Schmidt-Horning 2013; Warner 2003). This emphasis on recording and production technologies and related practices is understandable, given the acknowledged importance of technology for popular music genres. Yet when focusing on commercial records, attention inevitably turns to renowned artists and the work of audio engineers and producers. That leaves behind the majority of amateur, semi-professional and professional musicians who are not in the international spotlight. Furthermore, while the study of production involves the analysis of technology, it favours recording and processing technology such as microphones, mixing consoles and signal processing effects over source technology: the musical instruments where sound and expression begin. In light of the pivotal role of musical instruments in the creation of music, it is surprising that they are still a relatively unresearched field in popular music.

Given the vast complexity of musical instruments as cultural artifacts, and their fundamental importance to the making of music, it is worth pondering why they have been given relatively little attention in the study of music, and of popular music in particular ... For all too many popular music scholars, musical activity does not exist for all intents and purposes before the moment of recording. Such an assumption, whether explicit or unspoken, leaves scholars to concentrate upon a range of issues that, while of key importance, tend to exclude the ways in which instruments figure into musical practice and production. (Waksman 2003: 252) 
This statement in no way suggests that research on musical instruments in popular music does not exist. There are numerous examples of authors who have written about the electric guitar. Waksman (1999) explores the historical and cultural significance of the electric guitar by focusing on how influential performers have shaped the instrument's use and meaning. Herbst (2016) analyses the musical and cultural significance of guitar distortion for rock and metal music, and Uimonen (2016) discusses celebrity guitars as luxury items. Théberge (1997) deals with the increasing commodification of music-making and examines democratisation processes through advances in digital keyboard technology. From practitioners' perspectives, Bruford (2018) analyses how expert drummers experience creativity in performance. Recently, Brennan (2020) provided a long-overdue social history of the drum kit. There are also handbooks on popular music instruments (Bacon 1996), histories of manufacturers and inventors (Maloof 2004) and lists of the 'greatest players' of certain instruments, which often include information about their equipment and its influence on playing and sound (Kitts \& Tolinski 2002). Magazines such as Premier Guitar, Modern Drummer or Keyboard Magazine regularly feature so-called 'rig rundowns' that give insights into the secrets of renowned players' distinct tones. Gear reviews also take up large parts of these magazines. Academic, journalistic and educational resources focus on a range of topics, including various histories, inventions, star performers, vintage gear and the latest technology. In these discourses, the focus is mainly on musical and aesthetic issues but less so on the cultural significance and social relevance of musical instruments. Yet such a view is too limited and does not consider the multitude of meanings:

'Reading the instrument' ... involves acknowledging the complex relationship
between musical and extramusical factors in the cultural life of musical instru-
ments. This pursuit, in turn, involves thinking through the ways in which a
musical instrument becomes embedded in a given cultural setting, or alter-
nately in a web of discursive meanings that coats the materiality of the instru-
ment with a residue of symbolic import. (Waksman 2003: 252)

Musical instruments are deeply intertwined with cultural practices rooted in society. In the more direct sense, playing an instrument requires consideration of genre conventions and aesthetics. That may involve selecting the right instrument model, combining it with suitable effects and amplifiers, and fine-tuning the resulting sound within the arrangement of a larger ensemble. All these decisions, explicit or implicit, are based on musical traditions that shape the actions. Players must give thought to conventions they wish to follow and the importance of having an original sound. It is not only a question of tone; gear choice also affects playing feel, and thus, how emotions are expressed musically. Therefore, lack of access to the right equipment can hold performers back and prevent them from reaching their full potential. At least, this is what discussions in online message boards, musicians' magazines and equipment-related videos often suggest. 
With the availability of a wide range of music magazines, blogs, social media communities and message boards, musicians can easily keep up with the latest trends, which increases the likelihood that they will reflect on their musical setup and compare it to that of their fellow musicians. These media have become part of everyday life and tempt players more than ever to focus on their hobby's material side. According to Théberge (1997: 245), modern musicians do not necessarily consume more equipment than older generations of musicians, but 'consumption has become an integral aspect of their musical production practice'. Dealing with gear is part of modern musical practice, and its implications go beyond the mere activity of music-making.

Instruments are commodities, the sale of which represents an often unrecognized aspect of the business of music. They are material objects subject to variations in design, and often tied to broader shifts in the technological basis of music making ... Musical instruments are sources of knowledge, the material embodiment of musical theory and technique. They are cultural resources that can be used to transmit long-held traditions or to enact far-flung innovations. (Waksman 2003: 252)

The primary intentions for using instruments are music-related and require respective deliberations, decisions and actions. However, we argue that there are deeper cultural, social and psychological motives at work. Dealing with instruments necessarily involves broader attitudes, such as openness to innovation versus traditionconsciousness, which are part of a player's identity. Empirical research has even indicated that personality characteristics differ between musicians of various instruments (Bell \& Cresswell 1984; Cameron et al. 2015; see also Rötter \& Steinberg 2018). Although such research has focused mainly on personality traits like extraversion, everyday experience suggests that keyboard players, for example, would appreciate technological innovation more than guitarists who are more likely to use vintage guitars and analogue valve amplifiers (Herbst 2019b).

Consumption and collecting are two important practices associated with the broader socio-cultural networks linked to music-making. Both have been widely discussed in social sciences and cultural studies research focused on leisure, demonstrating how recreational activities such as making music are relevant to a person's identity (Stebbins 2009). Playing an instrument and acquiring gear touches on phenomena such as nostalgia and the 'extended self' (Belk 1988), which are influenced by sociodemographic factors like gender, age, relationship status and living situation (Belk 1995a). Less often discussed in the field of popular music technology are consumer research, marketing and business studies, all of which can provide a different perspective on the social context of consumption around making music. Such research offers theoretical approaches to understanding how 'desire' and 'necessity' (Braun et al. 2016), 'fetish' (Fernandez \& Lastovicka 2011), 'taste' (Arsel \& Bean 
2013), 'facilitation' (Hartmann 2016) and 'use-value' (Cole 2018) can nourish a musician's urge to invest in equipment. This economic view is one way of understanding buying behaviour in a broader socio-cultural context. It draws connections between musical practice and concepts such as 'craft consumption' (Campbell 2005), which describe the players' modification and customisation of stock items as a strategy for re-appropriating standard goods to shape their creative identity and obtain unique tools for musical expression.

The last fact worth mentioning is that the phenomenon is not limited to music. GAS occurs in many areas of everyday life. In music, it can be observed in hi-fi audio culture (Schröter \& Volmar 2016), record collecting (Shuker 2010), home recording (Strong 2012) and jingle composition (Fisher 1997). Outside music, GAS occurs amongst photographers (Arias 2013; Kim 2012; Sarinana 2013), aquarium hobbyists (Wolfenden 2016), amateur astronomists (Chen \& Chen 2017), cyclists (Peters 2013) and eBayers (Zalot 2013). Despite its high prevalence in contemporary culture, the phenomenon has hardly been researched. This book's overarching aim is to explore GAS in popular music from various perspectives, such as music technology, social, leisure and cultural studies, as well as consumption research, and to develop a theoretical understanding of this phenomenon (chapters 2 to 5). With original quantitative and qualitative empirical research, this working theory will be tested and refined (chapters 6 and 7). Some of the guiding questions are:

- Which sociodemographic variables and other personal, social and musical motives play a role in instrument consumption?

- Do players of various instrument types differ in their buying and collecting behaviour? Do musicians of electric or electronic instruments show a greater tendency towards GAS than those of acoustic instruments?

- What are the mental processes musicians are going through when the desire for music equipment develops?

- How do offline and online practices contribute to a pronounced interest in musical gear and the urge to buy?

The findings will provide insights into socio-cultural practices of how musicians deal with gear and what attitudes they have towards equipment. That will set the foundation for an interdisciplinary theory of the Gear Acquisition Syndrome grounded in empirical data. Lastly, it will be discussed how the psychological urge to acquire gear, consumption as part of music-making, and collecting are related.

GAS is assumed to occur in all musical genres, classical and popular, and concerns all instruments. It is not only common among players of traditional instruments but also widespread in modern, digital production-based forms of music-making, whose creators are tempted to invest in microphones, controllers and virtual plugins 
(Bourbon 2019; Carvalho 2012; Cole 2011; O'Grady 2019). Given the significant differences between all these groups and the respective activities, it is necessary to limit the research scope. While the theoretical chapters (2 to 5) can also be applied to classical musicians and music producers to some degree, we focus on players of popular music in the sample population of the empirical studies (chapters 6 and 7). More specifically, we will focus on the traditional core instruments of popular music genres that still rely on band instruments: guitar, bass, drums, keyboards, as well as saxophone (woodwind) and trumpet (brass), as exemplarily selected wind instruments.

\section{Book Structure}

\section{Chapter 2: Gear Acquisition Syndrome}

The Gear Acquisition Syndrome is a phenomenon with which musicians are commonly confronted. This chapter examines musicians' different attitudes to identify the reasons as to why many of them feel the urge to acquire new equipment. First, it gives an account of the so-called 'GAS attack', which is frequently described in blogs and other journalistic media. What triggers the musicians' ceaseless quest to improve their setup and drives them to acquire gear is discussed. The chapter finishes with the differentiation between various subgroups of musicians such as players, collectors, gear heads, purists and crafters. These groups are believed to have different attitudes towards equipment and diverging consumption patterns and manifestations of GAS.

\section{Chapter 3: Role and Context of Technology for Music-Making}

Here, the role of technology for popular music in general and in relation to the different attitudes and practices of players of various instruments is explored. Vintage instruments are another subject of investigation. What are the reasons for their popularity, and is innovation in music instrument technology important? If so, what are the consequences for the music industry? By analysing special-interest books, we examine which topics industry and media consider relevant, and what role equipment and sound quality play for popular music instruments. The chapter ends with a discussion of gendered practices in music-making. We have come to conclude that the musical instruments industry has historically tended to consider female musicians less relevant as consumers of gear because they were generally regarded as relatively inexperienced in the use of music technology. Although there are signs of change, our investigation into manufacturers' practices suggests that relatively few female musicians receive prestigious signature instruments and that they are often overlooked in endorsement deals. 


\section{Chapter 4: Collecting}

Little is known about how collecting relates to GAS, as it is a largely unexplored area of popular music research. By drawing on theories and empirical studies from various disciplines such as sociology, consumption research, psychology and psychiatry, this chapter offers a multidisciplinary perspective to evaluate the blurry relationship between GAS and collecting. As a starting point, collecting is established as a widespread practice in Western societies, and its benefits to identity construction are considered. Collecting is understood as a social practice marked by social hierarchies that are formed by individuals who seek to distinguish themselves through their prestigious collections and valuable items. The psychological and social processes and circumstances of collecting are examined in its entire range from the harmless pastime to obsessive acquisition and hoarding.

\section{Chapter 5: Consumption}

Consumption, by definition, is at the heart of the Gear Acquisition Syndrome. Based on the large variety of empirically derived theories within the multidisciplinary field of consumption research, this chapter focuses on musicians' relationships to their possessions to determine how these become the 'extended self' of their identity. Leisure studies provide a useful lens for understanding the strong motivations that drive musicians in their amateur and semi-professional endeavours. Key terms for GAS, 'desire' and 'necessity', are theorised, attempting to explain why musicians like to be seduced by gear and why they do not even mind participating in the marketing efforts of music industries. Two other relevant concepts, 'prosumption' and 'craft consumption', are explored. The discussion includes fabrication, modification and combination of music equipment as standard DIY practices. We found two effective facilitators of desire and the impulse to buy new gear in the online phenomena 'eBaying' and the exchange on message boards.

\section{Chapter 6: Interviews and Survey of Musicians}

GAS has not yet been studied academically. This chapter deepens and tests the theoretical deliberations developed in the previous chapters. It begins with an explorative study of musicians interviewed in a music store. What follows is a standardised online survey taking stock of musicians' gear collections, their attitudes and practices towards gear, their criteria for choosing an instrument and other personal, social and musical motives that affect their dealing with equipment. A large number of open comments help explain the survey results and reveal further practices not covered in academic and journalistic writing on GAS. 


\section{Chapter 7: Online Message Boards}

Musicians have always 'talked gear', but with the Internet, discussions have proliferated. Social media and special-interest message boards bring together people around the world who share the same interests. Building on the concept of 'communities of practice', we analyse how GAS is discussed in fifteen selected message boards. It is a common theme shaping community life. As a learned and expected behaviour, GAS is structurally reflected in recurring threads across all message boards. One of the main forms it takes is 'flipping', the selling of gear to buy other used equipment. Major GAS facilitators are eBay and other gear-related websites, besides the globally online operating musical instruments industry. The discourse focuses on the relationship between playing and interest in gear. Individual circumstances and personal motives explain varying interests in music equipment. Experimenting with gear is considered a natural part of learning and musical development. However, for various reasons, a musician might show more interest in gear than actually playing it: limited time, better compatibility with other responsibilities and family life, stagnation in musical development, lack of meaningful projects or artistic directions or plain boredom. Online discussions occasionally hint at impulsive and compulsive behaviour, suggesting that interest in gear is not always harmless. Speculations have it that GAS is a symptom of an underlying problem, so many discussions in the community revolve around how to counteract it. While there is general agreement that GAS is 'incurable', the discussions suggest several mitigating circumstances, strategies and principles that turn it into a harmless interest inseparable from music-making, with the potential to contribute to a musician's development.

\section{Chapter 8: Conclusion: Towards a Theory of GAS}

This concluding chapter takes stock of the theoretical and empirical insights into GAS. Even though the term emerged as 'Guitar Acquisition Syndrome', and specialinterest books easily create the impression that electric guitarists are most gear-centric and thus susceptible to GAS, this perception is not entirely accurate, as our findings show. The discrepancy between the thematic focus on gear in books about the electric guitar and the shared interest in equipment amongst all instrumentalists suggests that authors and publishers, perhaps even parts of the wider musical instruments industry, may have a distorted image. The chapter goes on evaluating the role of the Internet concerning the musicians' interest in gear and its temptation for GAS. It concludes that easy access to information, social exchange in special-interest communities and the large market for used instruments made available through auction and other selling websites are crucial factors for the prevalence of GAS in musicians' communities. GAS is a learned behaviour and expected in communities of practice. Fundamental to the urge to acquire gear is the indefinite quest to improve one's rig. GAS rarely disappears completely; it can strike in times of doubt, stagnation or other 
situations preventing musicians from playing or pursuing their projects. Like an umbrella, the term encompasses various practices related to the way musicians think about and deal with equipment. There is no single form of GAS; instead, the term summarises a spectrum of cultural practices related to and part of music-making. Since GAS accompanies musical learning processes, exploring the gear's affordances should be considered a contributing and reflecting part of musical expertise, at least in popular music. GAS is usually not the 'disease' often described. As a constant companion of musicians, it is possibly a sign that engagement in music has not lost its importance for the individual. 